\title{
Interleukin-33 Promotes Cell Survival via P38 \\ MAPK-mediated Interleukin- 6 Gene Expression and Release in Pediatric AML
}

\section{Yiqian Wang}

Guangzhou Medical University https://orcid.org/0000-0001-5369-6636

Haibo Su

Guangzhou Medical University

Muxia Yan

Guangzhou Women and Children's Medical Center

Li Zhang

Guangzhou Women and Children's Medical Center

Quanxin Li

Guangzhou Medical University

Xiaoqiong Gu

Guangzhou Women and Children's Medical Center

Qing Gong ( $\sim$ 443292273@qq.com )

Department of Biochemistry and Molecular Biology, GMU-GIBH Joint School of Life Sciences, Guangzhou Medical University, Guangzhou, China

\section{Research}

Keywords: AML, IL1RL1, IL-33, p38 MAPK, IL-6

Posted Date: August 17th, 2020

DOI: https://doi.org/10.21203/rs.3.rs-59371/v1

License: (c) (1) This work is licensed under a Creative Commons Attribution 4.0 International License. Read Full License 


\section{Abstract}

\section{Background}

Acute myeloid leukemia $(\mathrm{AML})$ is a fatal disease characterized by an accumulation of immature myeloid blasts in the bone marrow (BM). Cytokine provide signals for leukemia cell to better survive in the BM microenvironment. Previously, we identified interleukin-33 (IL-33) as a stimulator of cell survival in human AML cell line and primary mouse leukemia cells. However, the mechanism on how IL-33 regulates AML activity is not well known.

Methods

Flow cytometry, ELISA and cytometric bead array (CBA) were used to investigate the expression of IL1RL1 and serum cytokine levels in AML patient samples and cell culture supernatant. The correlation of serum IL-33 and IL- 6 levels was calculated by Pearson R test. Annexin V staining was used to examine the apoptosis of AML cells. RT-PCR was used to measure IL-6 expression in leukemia cells.

Results

The cell surface expression of IL-33-specific receptor, IL1RL1, is elevated in BM cells from AML patients at diagnosis, and the serum level of IL-33 in AML patients is higher than that of healthy donor controls. Moreover, IL-33 levels are positively associated with IL-6 levels in pediatric patients with AML. In vitro, IL33 treatment increased IL-6 mRNA expression and protein level in BM and peripheral blood (PB) cells from AML patients. IL-33 inhibited cell apoptosis by activating p38 MAPK pathway using human AML cell line as well as AML patient samples. Finally, IL-33 activated IL-6 expression in a manner that required p38 MAPK pathway using clinical AML samples.

\section{Conclusions}

Taken together, we demonstrate that p38 MAPK pathway is important for IL-33-mediated anti-apoptotic process as well as cytokine expression and release in pediatric patients with AML.

\section{Background}

Acute myeloid leukemia (AML) is a heterogeneous disease characterized by specific chromosomal abnormalities, and it accounts for approximately $20 \%$ of pediatric leukemias. In the past few decades, there is incremental progress in curing pediatric AML through refined treatment regimens and improved supportive care [1]. Although event-free survival (EFS) rate for children with newly diagnosed AML ranges from $50 \%$ to $60 \%$, currently, there is no standard treatment for relapsed or refractory AML $[2,3]$. Thus, achieving better outcome remains an important issue for the therapeutic success for treating pediatric AML patients. 
The tumor microenvironment can affect the growth, survival and drug resistance of cancer cells $[4,5]$. Cytokines existing in tumor microenvironment are known to have profound effects in promoting cancer progression [6]. Dysregulation of the complicated cytokine network could create a pro-tumorigenic microenvironment. In AML, the most relevant microenvironment is within the bone marrow (BM) [7]. A critical role of the BM and the development of AML have been reported in different studies [8-11]. However, the relationship between cytokines in the BM and leukemia cell activity are not fully investigated. Interleukin 33 (IL-33) belongs to IL-1 family of cytokines, and participates in various biological activities, including inflammation, immune response and infection process [12]. Recent studies have identified IL-33 as an important regulator during the development of hematologic malignancies. For example, in chronic myeloid leukemia (CML) expressing BCR-ABL1, IL-33 increases the proliferation and help leukemia cells exert resistance to the inhibitor imatinib [13]. Of note, other reports suggest that IL-33 have an immunosuppressive effect by regulating immune cell activities in $\operatorname{AML}[14,15]$.

Previously, we found that IL1RL1 is highly expressed in leukemia cells expressing CBFB-MYH11 [16, 17]. Further, we demonstrated that exogenous IL-33 treatment inhibited apoptosis of primary mouse leukemia cells as well as human AML cell line, HL-60 [18]. Further investigation revealed that IL-33/IL1RL1 axis exerted the anti-apoptotic effect by potentially activating p38 MAPK pathway. In addition, we found that exogenous IL-33 promoted the mRNA expression of inflammatory cytokine, IL- 6 in mouse leukemia cells from in vitro analysis [18]. Based on these findings, we thus hypothesized that IL-33-mediated p38 MAPK activation led to the induction of IL-6 expression and production, eventually protecting leukemia cells from apoptosis.

In the present study, we show that both IL-33 and IL1RL1 are upregulated in AML patient samples as compared healthy donors. By treating primary AML samples with IL-33 and anti-IL-33 antibody, we found that IL-33 led to IL-6 expression and production. In consistent to our previous study using AML cell line, we also confirmed that IL-33 inhibited apoptosis via activating p38 MAPK pathway by using AML patient samples. Finally, we found that the inhibition of p38 MAPK signaling abrogated the upregulation of IL-6 expression and secretion mediated by IL-33 in BM cells collected from primary AML samples. Collectively, our data demonstrate that IL-33 is capable of supporting AML cell survival as well as stimulating cytokine production via p38 MAPK pathway in pediatric patients with AML.

\section{Materials And Methods}

\subsection{Patient samples}

Freshly collected or frozen bone marrow (BM) or peripheral blood (PB) samples were obtained from AML patients or healthy donors in accordance with the Declaration of Helsinki involved in this study, and was approved by the Guangzhou Women and Children Medical Center Ethics Committee (ethics number: 
2020-39500). All patients were risk-classified according to the latest World Health Organization (WHO) classification $[19,20]$.

The medical history of AML patients and healthy control subjects was obtained, and all patients provided written informed consent. Mononuclear cells (MNCs) from PB and BM samples were obtained using Human Mononuclear Cells Separation Medium 1.077 (Dongfang Huahui) and used for subsequent experiments. Normal samples correspond to steady-state serum and BM samples from healthy donors were collected after informed consent. Information of AML patients and healthy donors was provided in Table 1.

\subsection{Tissue culture}

For in vitro study, both IL-33 (Sino Biological) and anti-IL-33 antibody (Biovision) were used at 100 $\mathrm{ng} / \mathrm{mL}$, and SB203580 used at $2 \mu \mathrm{M}$. The human AML cell line HL-60, BMMCs, and PBMCs were grown in RPMI1640 (ATCC) supplemented with 20\% FBS, 2mM L-Glutamine, and 1\% penicillin-streptomycin. All cells were grown at $37^{\circ} \mathrm{C}$ and $5 \% \mathrm{CO}_{2}$.

\subsection{ELISA and CBA assay}

Enzyme-linked immunosorbent assay (ELISA) (4A Biotech) was performed on cell culture supernatant and serum in AML patients and the healthy donors according to the manufacturer's protocol. Briefly, the samples and the standard samples were incubated with horseradish peroxidase (HRP)-conjugated antibodies. Substrate TMB was added which became blue under the catalytic action of peroxidase. Absorbance was measured at $450 \mathrm{~nm}$. The specific concentrations were calculated by using the software program CurveExpert version 1.4.

The in vitro IL-6 levels were evaluated in the collected supernatants using a cytometric bead array (CBA) for human IL-6 cytokine (BD Biosciences). The data were acquired using a CytoFlex S cytometer (Beckman Coulter) and analyzed by CytExpert 2.3 software (Beckman Coulter).

\subsection{Quantitative RT-PCR analysis}

Total RNA was isolated from cells using AG RNAex Pro Reagent (ACCURATE BIOTECHNOLOGY) according to the manufacturer's specifications, synthesized into CDNA using reverse transcription PCR (ACCURATE BIOTECHNOLOGY). Real-time quantitative analysis (qRT-PCR) was performed using SYBR Green Premix Pro Taq HS qPCR Kit (ACCURATE BIOTECHNOLOGY) with specific primers for Actb, and IL-6 (ACCURATE BIOTECHNOLOGY). The real-time PCR was run on a LightCycler ${ }^{\circledR} 96$ Instrument (Roche).Expression levels of genes were normalized to Actb mRNA. The 2- $\Delta \Delta \mathrm{CT}$ method was used to compare the relative expression levels of mRNA among different groups. Primers used for quantitative PCR include IL-6f: 5'- TGAACTCCTTCTCCACAAGCG -3' and IL-6r: 5'- TGGAATCTTCTCCTGGGGGTA -3'. Actbf:5'-GGATGCAGAAGGAGATCACTG -3' and Actbr.5'-CGATCCACACGGAGTACTTG -3') [21, 22].

\subsection{Western blot analysis}


Total proteins were extracted with the SDS lysis buffer (Beyotime) or RIPA buffer (KeyGEN). Samples were subjected to electrophoresis in SDS-PAGE gels and then transferred to a PVDF membrane (Millipore) for antibody blotting. The membrane was incubated with the primary antibody overnight, followed by 1hour incubation with horseradish peroxidase (HRP)-conjugated anti-rabbit or anti-mouse secondary antibodies at room temperature, washed and incubated with chemiluminescence (ECL) reagent (Beyotime). The following primary antibodies were used for western blotting: anti-p-p38 MAPK (Immunoway), anti-p38 (Immunoway), anti-pATF2 (Immunoway) and GAPDH (Abways).

\subsection{FACS analysis}

Primary samples were stained with APC conjugated anti-IL1RL1 (BD Biosciences). For the measurement of apoptosis, we used the FITC-conjugated Annexin V (Procell). Cells were analyzed using CytoFlex $S$ (Beckman Coulter) within 1 hour. Cell cycle was determined by propidium iodide (PI) staining after cells were fixed with ice-cold $70 \%$ ethanol for at least 30 minutes at $4^{\circ} \mathrm{C}$. FACS data was analyzed by CytExpert 2.3 software (Beckman Coulter) and ModFit software (Verity Software).

\subsection{Statistical analysis}

P-values and Pearson's correlation coefficients were calculated by Prism 8.0.1 (GraphPad software Inc.). The statistical significance of differences in mean values was analyzed by paired $t$ test. One-way ANOVA with Tukey post hoc test was used to perform multiple comparisons between experimental conditions. Data are represented as mean values \pm standard deviation. $P$ values $<0.05$ were considered statistically significant.

\section{Results}

\subsection{IL1RL1 expression and serum IL-33 level are higher in patients with AML}

To address the question whether IL1RL1 cell surface expression is upregulated in primary AML samples, we isolated MNCs from the BM of AML patients ( $A M L$ cohort; $n=5$ ) as well as healthy donors (HD cohort; $\mathrm{n}=8$ ), which were then stained with anti-IL1RL1. Flow-cytometric analysis revealed substantial IL1RL1 expression from most AML patients, while samples from healthy donors were expressing this receptor at very low levels (Fig.1A,B). By further analyzing the IL-33 profile in the serum collected from AML patients (AML cohort; $n=16$ ) at diagnosis and from healthy donors (HD; $n=9)$. We found that IL-33 levels were significantly elevated in AML patients compared with normal controls (Fig.1C). Importantly, in the patient group, the concentrations of IL-33 were elevated in a subset of AML patient samples, indicating that the variability may provide the ability to evaluate for potential relationships between IL-33 levels and clinical outcomes. Taken together, these results suggest that increased serum level of IL-33 along with the overexpression of IL1RL1 receptor in the BM could be a plausible mechanism for AML cells to survive.

\subsection{Baseline concentration of IL- 6 is positively correlated with IL-33 in the serum and IL-33 induces IL-6 expression in primary AML cells}


Previously, we demonstrated that exogenous IL-33 increases the expression of IL- 6 using primary mouse leukemia cells expressing $C b f b-M Y H 11$, which implies that a unique microenvironment is required to support leukemia cell growth[18]. To further elucidate the cytokine milieu associated with IL-33 in AML patients at baseline, we measured the levels of IL-33 with an ELISA kit and IL-6 with the CBA-based assay. We found that the serum concentrations of IL- 6 showed a significantly positive correlation with IL-33 levels at baseline ( $r=0.832, p=0.005)$ (Fig.2A). Next, to examine whether IL-33 could provoke enhanced IL6 expression in primary AML samples, we treated BM and PB cells with IL-33 or in combination with antiIL-33 antibody for 72 hours in culture and determined IL- 6 expression. We found that both IL-6 mRNA expression and secretion were elevated in response to IL-33, while antibody blockade of the IL-33 abrogated the enhancing effect of IL-33 on IL-6 expression and secretion in both BM and PB (Fig. 2B,C). Thus, our data suggest that IL-33 might work with IL-6 in the local and systemic circulation to help leukemia cell survive. Importantly, IL-33/IL1RL1 axis also stimulates cytokine production in patients with AML.

\subsection{IL-33 activates p38 MAPK pathway in primary AML samples}

IL-33 is found to be able to induce p38 MAPK pathway in both human natural killer (NK) cells and macrophages $[23,24]$ In addition, our previous work demonstrated that IL-33 increases the phosphorylation of p38 MAPK, which was inhibited by p38 MAPK inhibitor, SB203580 (SB) in human AML cell line HL-60 [18]. To further investigate this, we treated HL-60 cells with IL-33 alone or in combination with SB in culture. We then measured the phosphorylation levels of p38 MAPK and activating transcription factor (ATF2, Thr69/71), one of the downstream molecules of the MAPK signal pathway. We found that IL-33 significantly increased the phosphorylation of both p38 MAPK and ATF2 as compared to the untreated cells, while SB significantly reduced IL-33-induced p38 MAPK and ATF2 phosphorylation (Fig.3A,B). To determine if IL-33 activates p38 MAPK pathway in AML patient samples, primary AML cells from BM and PB were cultured and treated with IL-33 alone or in combination with SB for 72 hours. Phosphorylation of p38 MAPK and ATF2 were measured by western blot. We found that IL33 treatment stimulated the phosphorylation of both P38 MAPK and ATF2, whereas the addition of SB diminished the effect of IL-33-induced activation of p38 and ATF2 (Fig3.C-F). These results indicate that IL-33 can enhance the activation of p38 MAPK pathway in primary AML cells from both BM and PB. The data imply that IL-33-mediated p38 MAPK activation may be required by AML cells for their survival.

\subsection{IL-33 promotes cell survival via p38 MAPK pathway in primary AML samples}

To test whether IL-33 influences cell survival via p38 signaling in primary samples, we treated BM and PB cells from AML patients with IL-33 or SB alone, or in combination in culture for 72 hours and measured apoptosis and cell cycle status. We found that treatment with IL-33 led to a significant decrease in apoptosis, as compared to the untreated cells in both BM and PB, while SB blocked the anti-apoptotic effect mediated by IL-33 in BM. The combination of IL-33 and SB caused a trend towards higher apoptotic level as compared to IL-33 treatment alone in PB, although this was not statistically significant (Fig4.A-D). 
By performing cell cycle analysis using BM samples, we found that IL-33 alone caused a significant increase in cells in S phase as well as a significant decrease in G0/G1 phase, as compared to the untreated control, implying that IL-33 can induce proliferation to help cell survive in AML samples. Moreover, the combination of IL-33 and SB increased the proportion of cells in S phase as observed with IL-33 alone. Interestingly, we found that there was a statistically significant decrease in cells in G0/G1 phase in IL-33-treated cells, as compared to the untreated cells (Fig.4E). These results are consistent with our earlier work showing that IL-33 inhibits apoptosis via activating p38 MAPK and upregulates the percentage of cells in S phase in primary mouse leukemia cells and human AML cell line $[16,18]$. Together, our data indicate that IL-33 promotes cell survival by stimulating p38 MAPK pathway in AML patient samples.

\subsection{P38 MAPK contributes to IL-33-induced IL-6 expression in primary human samples}

Having demonstrated that IL-33 has a pro-survival role in AML samples by activating p38 and provoking IL-6 expression, we hypothesized that IL-33 may activate IL- 6 expression in a manner that requires p38 MAPK activation. To test this, we treated BM-MNCs from AML patients with IL-33, SB alone or in combination in culture for 72 hours and isolate RNA for qRT-PCR analysis. We observed that there was a significantly increased expression of IL-6 in IL-33-treated cells while SB cased a significant decrease in IL6 mRNA expression, as compared to the untreated (Fig.5A). Cells with the combination of IL-33 and SB showed a trend towards decreased IL- 6 mRNA expression compared to those with L-33 treatment alone, although this difference was not statistically significant. This result indicates that IL-33 enhances cytokine expression in primary human AML cells. We next sought to examine whether IL-33 could enhance IL- 6 release by leukemia cells using the same patient group. We measured the levels of IL- 6 with CBA-based assay after treating BM samples as described above for 72 hours. We observed that IL-33 significantly increased IL-6 release as compared to the untreated cells, whereas SB treatment diminished the enhancing effect of IL-33 on IL-6 secretion (Fig.5B). These results led us to conclude that P38 MAPK pathway is necessary for IL-33 to induce IL- 6 expression and release by leukemia cell from primary AML BM samples.

\section{Discussion}

Over the past decades, there has been a significant improvement in treating pediatric AML [25]. However, the outcomes of pediatric AML prognosis still remains unsatisfactory, and around $30 \%$ of patients relapse [26]. Therefore, novel therapeutic approaches to treat childhood AML are required. In recent years, a critical role of cytokines during AML development has been largely identified [27, 28]. IL-33 is a recently identified cytokine that belongs to IL-1 cytokine family [12]. Previously, we showed that IL $1 R L 1$, the receptor of IL-33, is highly expressed in leukemia cells expressing Cbfb-MYH11, and exogenous treatment with IL-33 promoted the survival of both primary mouse leukemia cells and human AML cell lines in vitro $[16,18]$. Further investigation revealed that IL-33/IL1RL1 axis can inhibit leukemia cell apoptosis via phosphorylating p38 MAPK [18]. However, so far, no clinical studies have been reported to demonstrate the role of IL-33/IL1RL1 axis in pediatric AML development. Thus, in this study we further examined the 
expression of IL1RL1 and IL-33 levels based on clinical data, and the mechanism of IL-33-mediated p38 MAPK stimulation in the maintenance of AML from pediatric patient samples.

By performing ELISA assay, we first showed that the IL-33 receptor IL1RL1 was constitutively expressed in BM cells from pediatric AML patients at diagnosis, whereas BM cells from healthy controls do not appear to express IL1RL1. In accordance with this observation, we found that there was an elevated level of IL-33 in serum from AML samples, as compared with HD cohorts. These results imply that IL-33 may play an important role in maintaining AML growth. Since accurate risk stratification is critical for

determining the intensity of therapy, our data generate the question of the relationship between IL-33 and prognosis in AML patients. However, due to the lack of intensified clinical data, we could only assume that serum IL-33 level represents a predictor of poor outcomes at least in subsets of AML patients. Thus, precisely tracking individual AML patients would provide additional information and better delineate the correlation of IL-33 levels and clinical outcomes.

Our previous work suggests that IL-33 leads to a decrease in apoptosis by potentially stimulating p38 MAPK pathway in human AML cell line HL-60 [18]. Here, we have extended this finding by demonstrating that IL-33 increased the phosphorylation of both p38 and ATF2 in primary AML samples, which assisted in cell survival. Importantly, the addition of p38 MAPK inhibitor significantly inhibited the phosphorylation of p38 and ATF2, which simultaneously abrogated IL-33 inhibition of apoptosis. Of note, p38 MAPK inhibitor reduced the expression and release of IL- 6 stimulated by IL-33. Therefore, our data indicate that p38 MAPK pathway is involved in IL-33-mediated anti-apoptotic effect in AML patient samples, potentially by stimulating IL- 6 expression and secretion. These works also imply that other p38 MAPKstimulated mediators, including ATF2 may also be important for the pro-survival effect of IL-33 in AML. In fact, ATF2 is a well-known stimulator of activator protein 1 (AP-1) transcription factors, both of which are involved in regulating cell cycle, proliferation and pathogenesis in the context of multiple diseases [29, 30]. By interacting with ATF2, one of AP-1 complex, c-Jun, has been found to play an important role during apoptosis progression in lymphocytic leukemia cells and participates in drug responses in $A M L$ cells $[30,31]$. Thus, it is conceivable that IL-33 selects p38-dependent downstream effectors to inhibit cell apoptosis, and stimulating the release of cytokine. Future direction should focus on investigating how p38 MAPK pathway is controlled by transcription factors including ATF2 and AP-1 complex, which could help in drug development.

\section{Conclusions}

Our results provide evidence that IL-33/IL1RL1 signaling may represent a novel cytokine-mediated mechanism contributing to AML development in pediatric patients. Further, our investigation supports a role for p38 MAPK pathway in AML maintenance and IL-33-induced cytokine release. Collectively, our work indicates that IL-33/p38 MAPK/IL-6 axis has the potential to be an effective treatment for pediatric AML patients.

\section{List Of Abbreviations}


AML: acute myeloid leukemia

AP-1: activator protein 1

ATF2: activating transcription factor 2

BM: bone marrow

CML: chronic myeloid leukemia

HD: healthy donor

HRP:horseradish peroxidase

IL1RL1: interleukin 1 receptor-like 1

IL-33: interleukin-33

MAPK: mitogen-activated protein kinase

PB: peripheral blood

SB: SB203580

\section{Declarations}

\section{Acknowledgements}

The authors would like to thank the Clinical Biological Resource Bank of Guangzhou Women and Children's Medical Center for providing all the clinical samples. This work was supported Department of Education of Guangdong Province (2019KQNCX114), Guangzhou Municipal Science and Technology Project (201904010067), Natural Science Foundation of Guangdong Province (2018A030313560, 2020A1515010009).

\section{Author Contributions}

Y.W., H.S., M.Y., X.G. and Q.G. designed the study; Y.W., H.S., and Q.L. performed experiments and analyzed the data; M.Y. collected clinical data; Y.W., H.S., M.Y., X.G. and Q.G. wrote the manuscript. All authors reviewed the manuscript in its final form.

\section{Consent for publication}

Not applicable.

\section{Conflicts of Interest}


The authors have no conflicts of interest to declare.

\section{References}

1. Rubnitz, J.E., et al., Minimal residual disease-directed therapy for childhood acute myeloid leukaemia: results of the AMLO2 multicentre trial. Lancet Oncol, 2010. 11(6): p. 543-52.

2. Gorman, M.F., et al., Outcome for children treated for relapsed or refractory acute myelogenous leukemia (rAML): a Therapeutic Advances in Childhood Leukemia (TACL) Consortium study. Pediatr Blood Cancer, 2010. 55(3): p. 421-9.

3. Ramos, N.R., et al., Current Approaches in the Treatment of Relapsed and Refractory Acute Myeloid Leukemia. J Clin Med, 2015. 4(4): p. 665-95.

4. Roma-Rodrigues, C., et al., Targeting Tumor Microenvironment for Cancer Therapy. Int J Mol Sci, 2019. 20(4).

5. Yuan, Y., et al., Role of the tumor microenvironment in tumor progression and the clinical applications (Review). Oncol Rep, 2016. 35(5): p. 2499-515.

6. Elsawa, S.F., et al., Comprehensive analysis of tumor microenvironment cytokines in Waldenstrom macroglobulinemia identifies CCL5 as a novel modulator of IL-6 activity. Blood, 2011. 118(20): p. 5540-9.

7. Wang, L.D. and A.J. Wagers, Dynamic niches in the origination and differentiation of haematopoietic stem cells. Nat Rev Mol Cell Biol, 2011. 12(10): p. 643-55.

8. Behrmann, L., J. Wellbrock, and W. Fiedler, Acute Myeloid Leukemia and the Bone Marrow Niche-Take a Closer Look. Front Oncol, 2018. 8: p. 444.

9. Lane, S.W., D.T. Scadden, and D.G. Gilliland, The leukemic stem cell niche: current concepts and therapeutic opportunities. Blood, 2009. 114(6): p. 1150-7.

10. Cogle, C.R., et al., Functional integration of acute myeloid leukemia into the vascular niche. Leukemia, 2014. 28(10): p. 1978-1987.

11. Isidori, A., et al., The role of the immunosuppressive microenvironment in acute myeloid leukemia development and treatment. Expert Rev Hematol, 2014. 7(6): p. 807-18.

12. Schmitz, J., et al., IL-33, an interleukin-1-like cytokine that signals via the IL-1 receptor-related protein ST2 and induces T helper type 2-associated cytokines. Immunity, 2005. 23(5): p. 479-90.

13. Levescot, A., et al., BCR-ABL-induced deregulation of the IL-33/ST2 pathway in CD34+ progenitors from chronic myeloid leukemia patients. Cancer Res, 2014. 74(10): p. 2669-76. 
14. Gao, X., et al., Tumoral expression of IL-33 inhibits tumor growth and modifies the tumor microenvironment through CD8+ T and NK cells. J Immunol, 2015. 194(1): p. 438-45.

15. Xiao, P., et al., Interleukin 33 in tumor microenvironment is crucial for the accumulation and function of myeloid-derived suppressor cells. Oncoimmunology, 2016. 5(1): p. e1063772.

16. Wang, Y., et al., IL $1 R L 1$ is dynamically expressed on Cbfb-MYH11(+) leukemia stem cells and promotes cell survival. Sci Rep, 2019. 9(1): p. 1729.

17. Hyde, R.K., et al., Cbfb/Runx 1 repression-independent blockage of differentiation and accumulation of Csf2rb-expressing cells by Cbfb-MYH11. Blood, 2010. 115(7): p. 1433-43.

18. Wang, Y., et al., IL-33/IL $1 R L 1$ axis regulates cell survival through the p38 MAPK pathway in acute myeloid leukemia. Leuk Res, 2020. 96: p. 106409.

19. Alessandrino, E.P., et al., WHO classification and WPSS predict posttransplantation outcome in patients with myelodysplastic syndrome: a study from the Gruppo Italiano Trapianto di Midollo Osseo (GITMO). Blood, 2008. 112(3): p. 895-902.

20. Arber, D.A., et al., The 2016 revision to the World Health Organization classification of myeloid neoplasms and acute leukemia. Blood, 2016. 127(20): p. 2391-405.

21. Midgley, A.C., et al., 17beta-estradiol ameliorates age-associated loss of fibroblast function by attenuating IFN-gamma/STAT1-dependent miR-7 upregulation. Aging Cell, 2016. 15(3): p. 531-41.

22. Poniewierska-Baran, A., et al., Human rhabdomyosarcoma cells express functional pituitary and gonadal sex hormone receptors: Therapeutic implications. Int J Oncol, 2016. 48(5): p. 1815-24.

23. Endo, Y., et al., The interleukin-33-p38 kinase axis confers memory Thelper 2 cell pathogenicity in the airway. Immunity, 2015. 42(2): p. 294-308.

24. Fehr, S., et al., Impact of p38 MAP Kinase Inhibitors on LPS-Induced Release of TNF-alpha in Whole Blood and Primary Cells from Different Species. Cell Physiol Biochem, 2015. 36(6): p. 2237-49.

25. Zwaan, C.M., et al., Collaborative Efforts Driving Progress in Pediatric Acute Myeloid Leukemia. J Clin Oncol, 2015. 33(27): p. 2949-62.

26. Lonetti, A., A. Pession, and R. Masetti, Targeted Therapies for Pediatric AML: Gaps and Perspective. Front Pediatr, 2019. 7: p. 463.

27. Han, Y., et al., Th17 cells and interleukin-17 increase with poor prognosis in patients with acute myeloid leukemia. Cancer Sci, 2014. 105(8): p. 933-42.

28. Stevens, A.M., et al., Interleukin-6 levels predict event-free survival in pediatric AML and suggest a mechanism of chemotherapy resistance. Blood Adv, 2017. 1(18): p. 1387-1397. 
29. Lopez-Bergami, P., E. Lau, and Z. Ronai, Emerging roles of ATF2 and the dynamic AP1 network in cancer. Nat Rev Cancer, 2010. 10(1): p. 65-76.

30. Watson, G., Z.A. Ronai, and E. Lau, ATF2, a paradigm of the multifaceted regulation of transcription factors in biology and disease. Pharmacol Res, 2017. 119: p. 347-357.

31. Min, B.W., et al., Transcription of the protein kinase C-delta gene is activated by JNK through c-Jun and ATF2 in response to the anticancer agent doxorubicin. Exp Mol Med, 2008. 40(6): p. 699-708.

\section{Tables}

\section{Table 1. Characteristics of AML patients collected in this study.}

Age is given in years at the time of initial diagnosis. Patients were assigned a risk group based on the World Health Organization (WHO) classification. Mixed lineage leukemia translocations are defined as poor risk factors. F, female; $\mathrm{M}$, male; $\mathrm{HD}$, healthy donor; $\mathrm{ND}$, not done: ITD, internal tandem duplication. 


\begin{tabular}{|c|c|c|c|c|c|}
\hline Patient number & Age/sex & Cytogenetics & Risk group & FAB type & Blasts,\% \\
\hline 1 & $2.3 / \mathrm{F}$ & $t(1 ; 3)$ & $\mathrm{HR}$ & M7 & 60 \\
\hline 2 & $13 / F$ & $-7 \rrbracket+1$ & $\mathrm{HR}$ & M5 & 35 \\
\hline 3 & $8 / F$ & Complex & LR & M4/M5 & 54 \\
\hline 4 & $5.1 / F$ & $\operatorname{add}(12)(\mathrm{p} 11.2), \operatorname{add}(19)(\mathrm{p} 13)$ & LR & M0 & 67 \\
\hline 5 & $14 / \mathrm{M}$ & $t(15 ; 17)$ & $\mathrm{HR}$ & M3 & 76 \\
\hline 6 & $3.8 / \mathrm{M}$ & FLT3-ITD & LR & M4 & 55 \\
\hline 7 & $5.8 / F$ & Normal & LR & M4/M5 & 87 \\
\hline 8 & $7 / F$ & $\operatorname{inv}(16), t(16,16)$ & LR & M4 & 44 \\
\hline 9 & $1.1 / \mathrm{M}$ & $t(9 ; 11)$ & $\mathrm{HR}$ & M2 & 55 \\
\hline 10 & $9 / \mathrm{M}$ & $t(15 ; 17)$ & LR & M3 & 86 \\
\hline 11 & $8 / F$ & $t(6 ; 11)$ & LR & M5 & 77 \\
\hline 12 & $2.6 / \mathrm{M}$ & $\mathrm{t}(9 ; 11)$ & $\mathrm{HR}$ & M5 & 70 \\
\hline 13 & $5.4 / \mathrm{M}$ & $t(10 ; 11)$ & $\mathrm{HR}$ & M5 & 86 \\
\hline 14 & $5.5 / F$ & $t(8 ; 21), 9 q 11$ del & $\mathrm{HR}$ & M2 & 88 \\
\hline 15 & $8 / \mathrm{M}$ & FLT3-ITD & $\mathrm{HR}$ & M2 & 37 \\
\hline 16 & $7 / \mathrm{M}$ & FLT3-ITD & LR & M2 & 55 \\
\hline 17 & $10 / \mathrm{M}$ & Normal & $\mathrm{HR}$ & $\mathrm{M} 4 / \mathrm{M} 2$ & 55 \\
\hline 18 & $2.4 / F$ & Complex, t(4:11) $₫+7 \rrbracket+22$ & LR & ND & 71 \\
\hline 19 & $3 / \mathrm{M}$ & FLT3-ITD & $\mathrm{HR}$ & M4 & 41 \\
\hline 20 & $12 / \mathrm{M}$ & Complex,$t(8 ; 21)$ & $\mathrm{HR}$ & M2 & 76 \\
\hline 21 & $7 / \mathrm{M}$ & $t(15 ; 17)$ & LR & M3 & 72 \\
\hline 22 & $2 / \mathrm{F}$ & -7 & $\mathrm{HR}$ & M2 & 22 \\
\hline 23 & $6.8 / \mathrm{M}$ & Complex,t(8;21),-X & $\mathrm{HR}$ & M2 & 70 \\
\hline 24 & $7 / \mathrm{M}$ & Normal & LR & M2 & 49 \\
\hline 25 & $1.8 / \mathrm{F}$ & Complex & $\mathrm{HR}$ & M2 & 41 \\
\hline 26 & $13 / \mathrm{M}$ & $t(9 ; 22)$ & $\mathrm{HR}$ & M2 & 96 \\
\hline 27 & $11 / F$ & $t(15 ; 17)$ & $\mathrm{HR}$ & M3 & 89 \\
\hline 28 & $1.2 / \mathrm{M}$ & Complex,t(1:22) & $\mathrm{HR}$ & M7 & 49 \\
\hline
\end{tabular}




\begin{tabular}{|c|c|c|c|c|c|}
\hline 29 & $7 / \mathrm{M}$ & Complex, t(15;17) & $\mathrm{HR}$ & M3 & 90 \\
\hline 30 & $3.5 / F$ & $\mathrm{t}(9 ; 11)$ & $\mathrm{HR}$ & M2 & 36 \\
\hline 31 & $/ F$ & +21 & LR & M3 & 86 \\
\hline 32 & $7 / \mathrm{M}$ & ND & LR & ND & 76 \\
\hline 33 & $1.2 / \mathrm{M}$ & ND & ND & MO & 53 \\
\hline 34 & $8 / \mathrm{M}$ & ND & ND & M2 & 50 \\
\hline HD1 & $2.1 / F$ & - & - & - & - \\
\hline HD2 & $3.5 / F$ & - & - & - & - \\
\hline HD3 & $3 / \mathrm{M}$ & - & - & - & - \\
\hline HD4 & $2.1 / \mathrm{M}$ & - & - & - & - \\
\hline HD5 & $3 / F$ & - & - & - & - \\
\hline HD6 & $2.9 / \mathrm{F}$ & - & - & - & - \\
\hline HD7 & $3.4 / \mathrm{M}$ & - & - & - & - \\
\hline HD8 & $3.5 / F$ & - & - & - & - \\
\hline HD9 & $3.6 /$ & - & - & - & - \\
\hline HD10 & $3.8 / \mathrm{M}$ & - & - & - & - \\
\hline HD11 & $6.3 / F$ & - & - & - & - \\
\hline HD12 & $1 / F$ & - & - & - & - \\
\hline HD13 & $4 / \mathrm{M}$ & - & - & - & - \\
\hline HD14 & $4.1 / \mathrm{M}$ & - & - & - & - \\
\hline HD15 & $10 / F$ & - & - & - & - \\
\hline HD16 & $11 / \mathrm{F}$ & - & - & - & - \\
\hline
\end{tabular}

Figures 
Figure 1

A
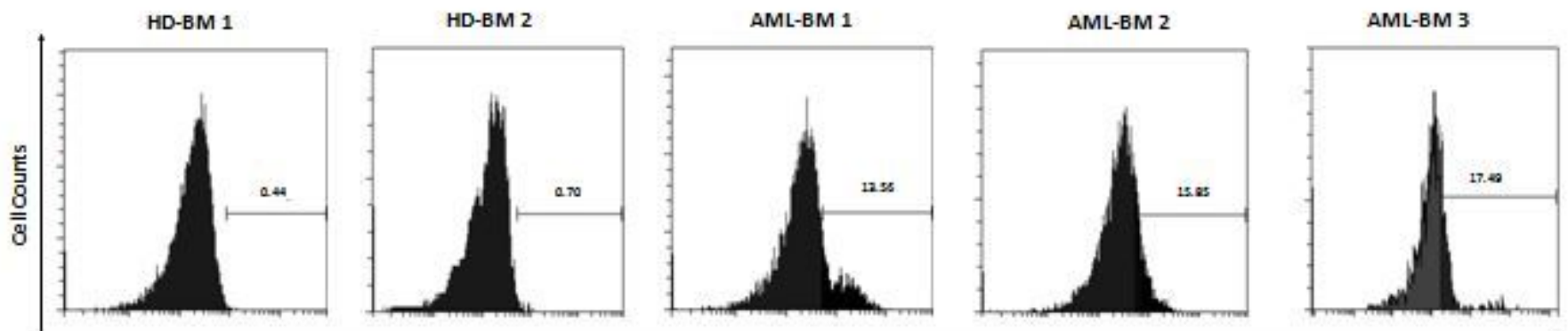

IL1RL1

B

C
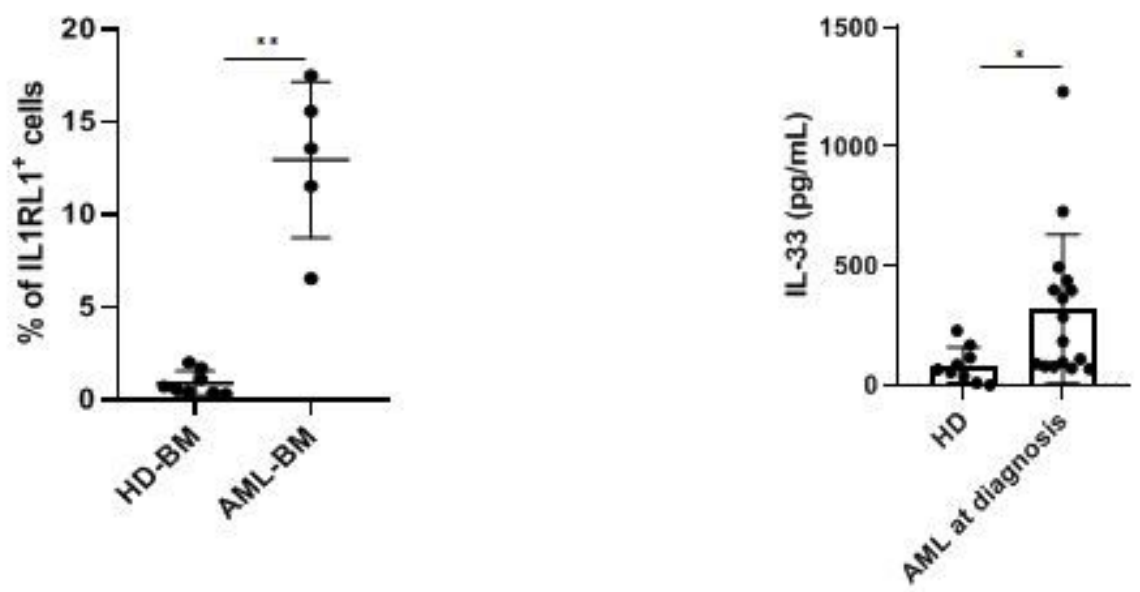

Figure 1

AML patients at diagnosis have elevated IL1RL1 expression and IL-33 level in the serum. (A-B) IL1RL1 expression is increased on the cell surface of BM cells from AML cohorts. BMMCs from patients with $A M L$ and healthy donors were analyzed for IL1RL1 expression. Each symbol represents one healthy donor or patient with AML. (C) ELISA assay was used to measure IL-33 levels in serum from AML patients at diagnosis and normal children. Each symbol represents one healthy donor or patient with AML. $N \geq 3$; ${ }^{*} \mathrm{P}<0.05 ;{ }^{*} \mathrm{P}<0.01$. Paired $t$ test. 
A

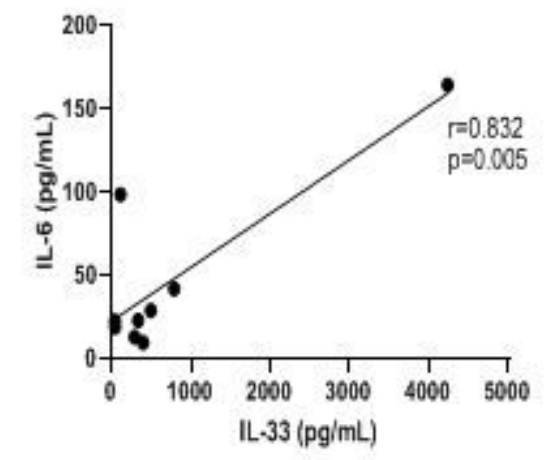

c

Bone marrow

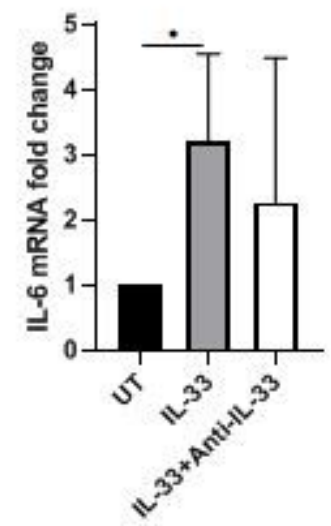

B

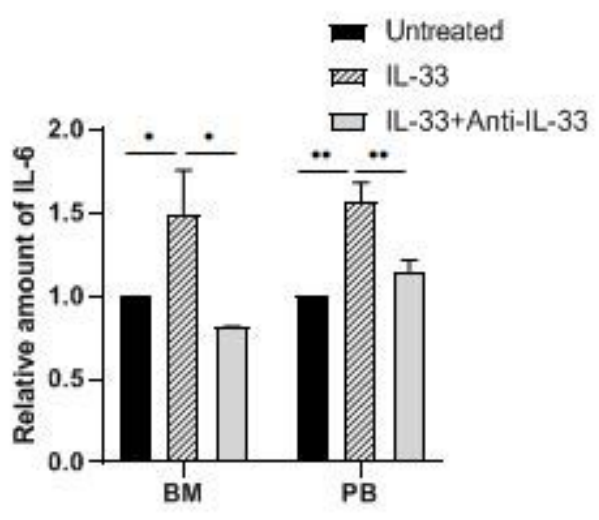
Peripheral blood

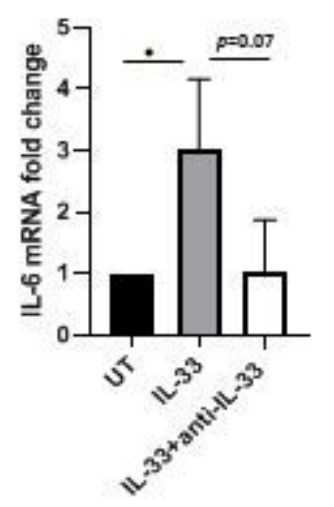

Figure 2

IL-33 induces IL-6 expression and secretion in primary AML cells. (A) The relationship between IL-33 and IL-6. Spearman rank correlation analysis was performed to evaluate the correlation of serum IL-33 with IL6 in patients with AML at diagnosis. (B) PBMCs and BMMCs from patients diagnosed with AML were incubated for 72 hours with IL-33 $(100 \mathrm{ng} / \mathrm{mL})$ or combined with anti-IL-33 $(100 \mathrm{ng} / \mathrm{mL})$. CBA was used to simultaneously measure IL- 6 in supernatants from cell cultures, and plotted as fold change compared with untreated samples. (C-D) RNA/cDNA expression of cells from (B) was analyzed using quantitative real-time PCR using Actb as a reference control. Data are plotted as relative gene expression compared with the untreated control. A significant difference in the mean IL-6 mRNA expression between compared with untreated cells was found in both $B M$ and PB samples. $N \geq 3 ; * P<0.05 ; * * P<0.01$. One-way ANOVA test. 
Figure 3

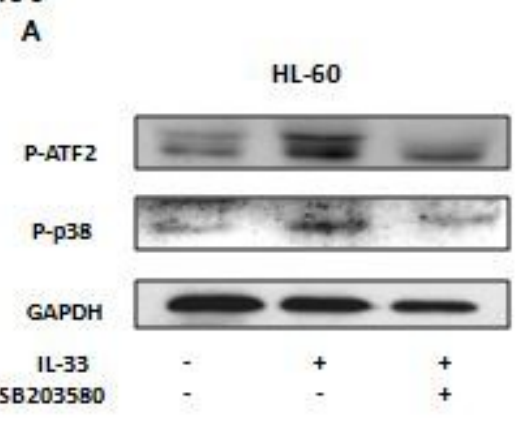

D

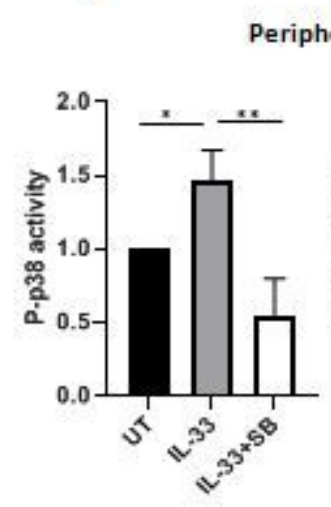

B
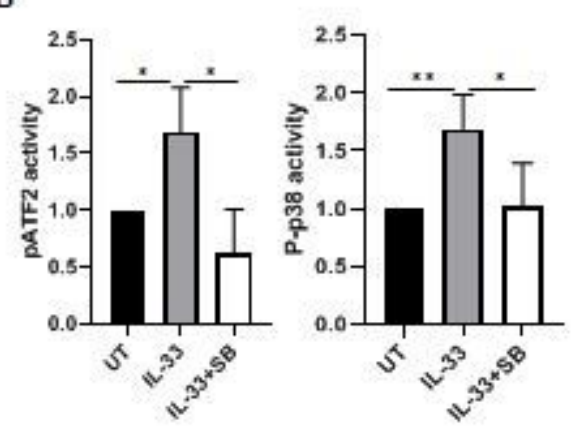

E

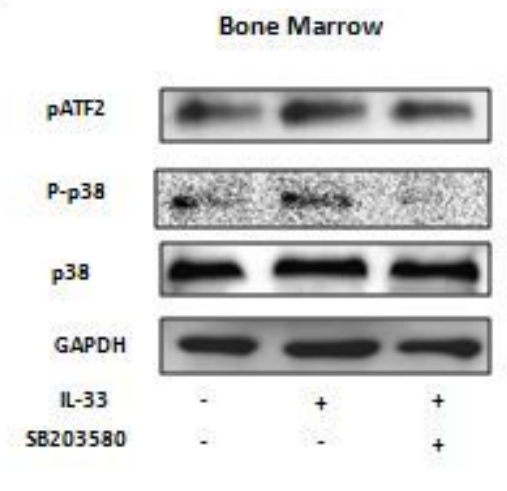

$\mathrm{C}$

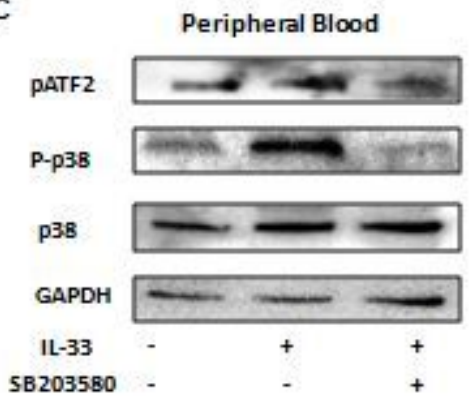

F

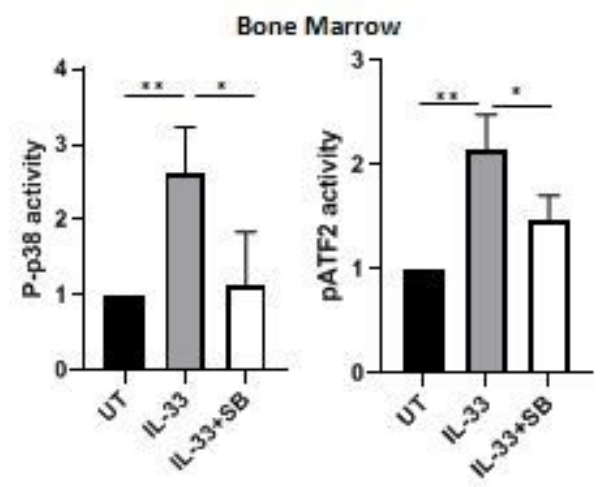

Figure 3

IL-33 activates p38 MAPK pathway in primary AML samples. (A-B) The AML cell line HL-60 were treated with IL-33 $(100 \mathrm{ng} / \mathrm{mL})$ or in combination with SB (20 uM). P-p38囚pATF2, or GAPDH protein expression was probed by western blot analysis. The bar graph shows the quantification of p-p38 and pATF2 protein in all groups. (C-F) PBMCs and BMMCs from patients diagnosed with AML were incubated for 72 hours with IL-33 $(100 \mathrm{ng} / \mathrm{mL})$ or in combination with SB $(20 \mathrm{uM})$. P-p38囚p38, pATF2, or GAPDH protein expression was probed by western blot analysis. The bar graphs show the quantification of $\mathrm{p}-\mathrm{p} 38$ and pATF2 protein in all groups. $\mathrm{N} \geq 3 ; * \mathrm{P}<0.05 ;{ }^{*} \mathrm{P}<0.05$. One-way ANOVA test. 
A

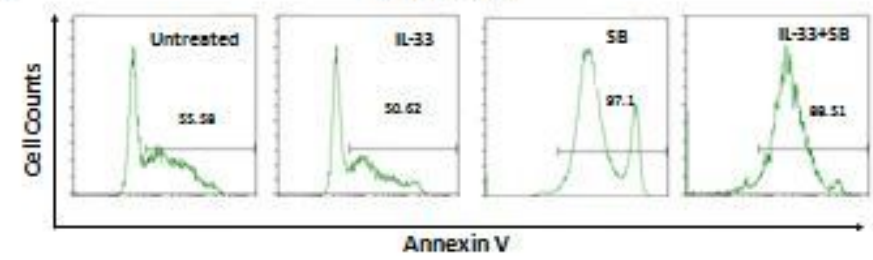

C

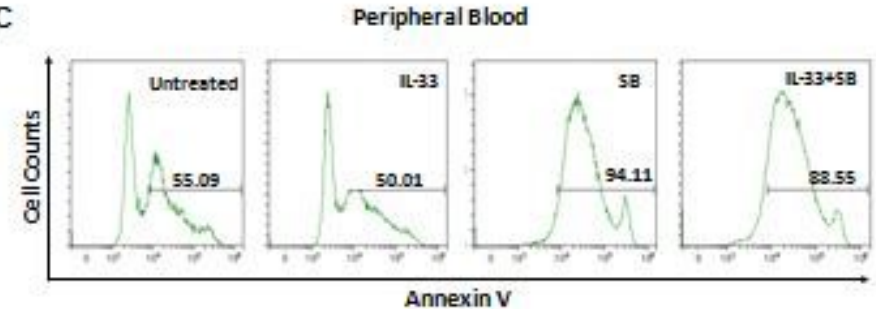

E

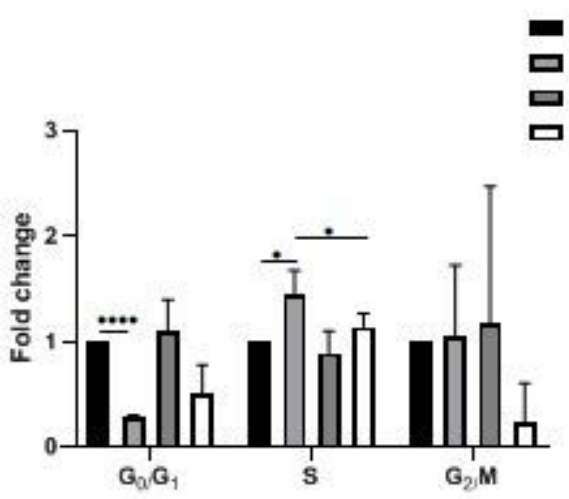

B
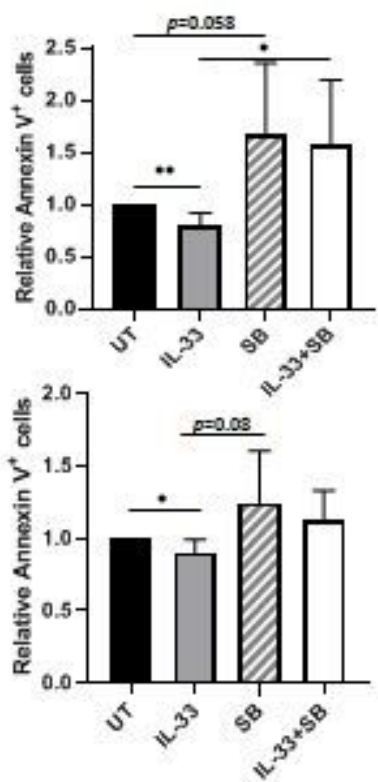

Figure 4

IL-33 promotes cell survival via p38 MAPK pathway in primary AML samples. BMMCs (A-B) and PBMCs (C-D) from pediatric patients with AML were cultured with IL-33 (100ng/mL), or SB (2 uM) alone, or in combination for 72 hours, and apoptosis was measured by Annexin $V$ staining. Bar graphs show the relative Annexin $V$ staining of leukemia cells as compared to the untreated cells. (E) BMMCs from AML patients were analyzed for cell cycle status. Bar graph shows the relative percentage of leukemia cells in the indicated phase of the cell cycle after culture for 72 hours in the presence of IL-33 (100 ng/mL), SB (20 uM) or in combination, compared to the untreated cells. $\mathrm{N} \geq 3 ;{ }^{*} \mathrm{P}<0.05 ;{ }^{*} \mathrm{P}<0.05 ;{ }^{*} * \star * \mathrm{P}<.0001$. Oneway ANOVA test. 
Figure 5

A

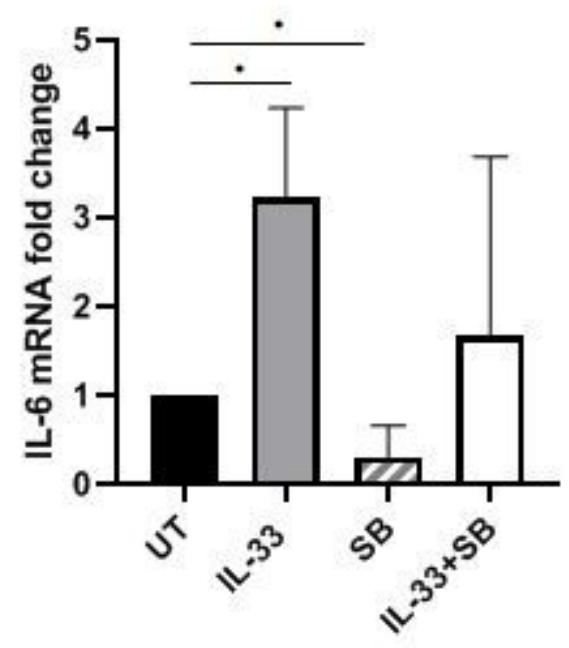

B

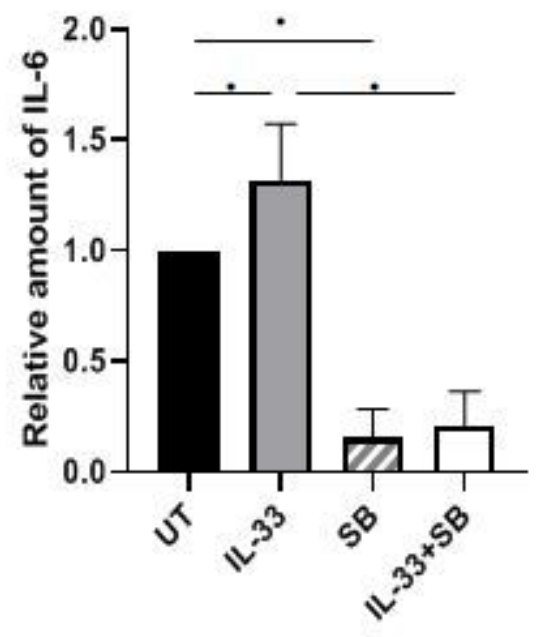

Figure 5

p38 MAPK pathway participates in IL-33-stimulated IL-6 expression and release. BMMCs from pediatric patients with AML were cultured with IL-33 (100ng/mL), or SB (2 UM) alone, or in combination for 72 hours. (A) RNA/cDNA expression of cells was analyzed using quantitative real-time PCR using Actb as a reference control. Data are plotted as relative gene expression compared with the untreated control. (B) CBA was used to simultaneously measure IL-6 in supernatants from cell cultures, and plotted as fold change compared with untreated samples. $N \geq 3 ; * P<0.05$. One-way ANOVA test. 